\title{
Philosophiques
}

\section{Philosophie du droit : théorie transcendantale et non-naturaliste. - Réponse à François Blais}

\section{Alain Renaut et Lukas K. Sosoe}

Volume 21, numéro 1, printemps 1994

URI : https://id.erudit.org/iderudit/027260ar

DOI : https://doi.org/10.7202/027260ar

Aller au sommaire du numéro

Éditeur(s)

Société de philosophie du Québec

ISSN

0316-2923 (imprimé)

1492-1391 (numérique)

Découvrir la revue

Citer cet article

Renaut, A. \& Sosoe, L. K. (1994). Philosophie du droit : théorie transcendantale et non-naturaliste. - Réponse à François Blais. Philosophiques, 21(1), 253-257.

https://doi.org/10.7202/027260ar d'utilisation que vous pouvez consulter en ligne. 


\title{
PHIIOSOPHIIE DU DROII : THÉORIE IRANSCENDANTALE ET NON-NATURALISTE. Réponse à François Blais
}

\author{
par \\ Alain Renaut et Lukas K. Sosoe
}

L'auteur de la recension de Philosophie du droit, il faut d'emblée le souligner, n'a pas tout à fait saisi l'objectif de l'ouvrage. L'occasion semble donc tout à fait propice de revenir sur les points qui méritent d'être pris en considération et qui révẻlent que $F$. Blais a négligé l'essentiel au profit de considérations purement scolaires et secondaires et qu'il n'a pas été tout à fait au niveau de sa tâche critique qui est demandée.

Au-delà du positionnement philosophique de l'auteur, s'il en a un, ce qui frappe dans la recension qu'a faite François Blais de Philosophie du droit, c'est le ton belliqueux; un ton inutilement agressif, du moins en l'absence de raison apparente. Qui plus est, l'investissement auquel a consenti l'auteur dans une critique aussi violente aurait pu servir à une lecture plus objective qui, au-delà des divergences, pourrait constituer un enjeu philosophique sérieux présentant un certain intérêt. Ce qui de toute évidence n'est pas le cas.

Le point central de la critique de François Blais tourne autour du positivisme juridique. Bien que l'ouvrage Philosophie du droit ne porte pas sur le positivisme juridique en tant que tel, la critique en fait l'objet essentiel. On y rappelle une évidence dont nous n'aurions pas tenu compte : Le positivisme juridique est une doctrine du droit qui affirme essentiellement qu'entre le droit positif et la moralité, il n'existe qu'un rapport contingent ». Pour l'auteur, le positivisme distingue le problème cognitif du droit de la question d'une théorie normative du droit. Bien que le positivisme trouve cette dernière question tout à fait légitime et n'exclut pas qu'on puisse s'interroger sur les conditions de possibilité même du droit ou sur sa légitimité, il ne la prend pas en considération et ceci pour des raisons méthodologiques, évidentes elles aussi. Raison suffisante, non pas pour défendre, contre la perspective qui est la nôtre, le positivisme juridique, mais uniquement pour « établir les faits »!

À suivre François Blais dont nous ne pensons pas déformer la pensée, le positivisme étant compatible avec une theorie jusnaturaliste, puisqu'il s'agit bien de deux perspectives différentes, l'élaboration d'une théorie du droit naturel ne nécessite nullement que soit rejeté, au moins critiqué, le positivisme ou alors qu'on attire l'attention sur ces limites quant à la pensée du droit naturel. Le faire serait tout au plus chercher un adversaire trop facile ou organiser 
une dispute qui n'a pas de raison d'être. Bref, selon l'esprit de la critique, et toujours pour des raisons méthodologiques, la «cohabitation pacifique » entre une philosophie normative du droit et une philosophie positiviste du droit est tout à fait concevable.

Accepter une telle critique, c'est quelque peu sous-déterminer l'exigence de reconstruction du droit naturel face aux différents positionnements philosophiques qui en rendent la pensée impossible; c'est, d'une part, réduire le problème du droit naturel et du positivisme juridique à une simple question de définition et d'autre part, déclarer que, depuis Platon aux philosophes contemporains, en passant par St. Augustin avec son fameux « Lex injusta lex non est $\gg$, l'opposition, sous toutes ses formes, entre le positivisme juridique et le jusnaturalisme, bref, tout ce que nous pouvons entendre par philosophie du droit, s'est occupé d'un faux problème que la seule distinction des deux perspectives méthodologiques aurait pu longtemps résoudre. En ce sens, le « retour éternel du droit naturel » dont parlait encore Rommen au lendemain de la deuxième guerre mondiale n'est, tout au plus, attribuable qu'à l'ignorance des deux perspectives dont l'une se réclame scientifique et l'autre morale. Le débat reste donc complètement dépourvu d'intérêt puisqu'il repose sur un malentendu que l'auteur entend trancher en attirant l'attention sur l'erreur qui lui est sous-jacente : le manque de prise en compte des deux perspectives analytiquement distinctes qui, selon lui, ne sembleraient entretenir, au plan formel, méthodologique, aucune relation l'une avec l'autre et au plan du contenu, qu'un rapport purement contingent.

Si la controverse entre le positivisme juridique et le droit naturel n'est, tout au plus, qu'un exercice scolaire stérile et sans aucun intérêt, pourquoi donc malgré cette distinction que personne ne semble avoir niée, F. Blais cherche-t-il à attirer l'attention sur les nouveaux aspects du débat qui, selon nous, n'a pas d'enjeu réel qui dépasse la « querelle du positivisme » telle qu'elle a été menée, dans la philosophie allemande d'une part, l'abondante discussion entre les positivistes et les jusnaturalistes qui, recommencée depuis la deuxième guerre mondiale, vient à peine de s'essouffler, du moins sur le continent? Qu'est-ce qui interdit que soit reposée la question de la légitimité des principes méthodologiques d'une théorie cognitive du droit qui prétend à la description, objective et neutre, d'un système juridique ou de certaines normes ? L'auteur aura au moins, sinon l'intelligence, du moins l'honnêteté de reconnaître, dans la remise en question de la prétendue neutralité que ce soit par le biais de la réception de la pensée wittgensteinienne, une stratégie de fondation du droit naturel non seulement chez Finnis, mais encore chez d'autres comme R. Dworkin qui, dans les parties qu'il convient d'appeler critique et constructive de son ouvrage : Taking Rights seriously (1977) rejette l'indépendance des deux perspectives pour une théorie du droit. Ce qui n'est pas une nouveauté puisque c'est en ces termes même que la question se pose. Si, comme F. Blais le dit à la suite de Weinreb, une fois la distinction de l'être et du devoir-être effectuée, le problème serait résolu, pourquoi, même dans la philosophie anglo-américaine que selon lui, nous ignorons parfaitement, y a-t-il eu, depuis Hume, Clark, Henry Sidgwick, G. E. Moore et Prior etc. un débat sur la question, connue dans la littérature sous le titre de «Is-Ought Problem », et dont la position de certains auteurs contemporains les plus représentatifs est résumée dans l'ouvrage de 
W. D. Hudson : Is-Ought Question, (New York, 1969), un ouvrage qui, il faut le souligner, est loin de constituer un résumé des perspectives en question? Pourquoi les Archives de Philosophie Juridique et Sociale (Archiv für Rechts- und Sozialphilosophie, 1968) auraient consacré un numéro spécial au problème du positivisme et du droit naturel sous le titre : Sein und Sollen im Erfahrungsbereich des Rechts (Être et devoir-être dans le domaine de l'expérience juridique) ? Pourquoi enfin Kelsen, après la définition de la perspective qui est celle de la Théorie pure $d u$ droit s'acharne-t-il contre la doctrine du droit naturel qu'il qualifie de métaphysique du droit? Par où l'on voit que ce qui apparaît ici comme une simple définition analytique qu'il serait nècessaire d'effectuer, repose en fait, sur un problème qui, selon les auteurs, se formule en terme d'autonomie de l'éthique et / ou du droit, en termes d'une possibilité de concevoir le droit sans le réduire au fait de l'existence d'un système juridique. Bref il s'agit de savoir si le droit n'inclut pas dans sa fondation dernière un élément qui n'est pas réductible au fait, à la volonté du législateur, au droit coutumier ou même à la force ou même à une simple exigence de déduction logique transcendantale du droit. Un problème dont on ne dispose pas facilement, si l'on connaît véritablement la littérature, moyennant une simple distinction de perspective. On admettra également que ces auteurs ne sont pas tous naïs au point de confondre une théorie cognitive du droit avec une théorie du droit naturel et sa fonction critique. Le manque de modestie et de simplicité a certainement empêché F. Blais de le remarquer.

Toutefois, ce n'était pas tant notre objectif de présenter le positivisme lou les positivismes) dans ses (ou leurs) récents développements que de nous interroger sur les conditions intellectuelles sans lesquelles le droit ne saurait retrouver sa fonction critique par rapport à tel ou tel système juridique. Cet objectif, qui entend analyser philosophique les différentes revendications faites au nom du droit, plus précisément au nom des droits de l'homme, et dont l'introduction de Philosophie du droit fait d'ailleurs amplement mention, semble avoir échappé à l'attention de l'auteur qui n' y a perçu qu'« une théorie substantielle des droits fondamentaux » dont il se demande comment elle pourrait affecter le droit. L'erreur qui consiste à « affirmer que l'existence et la reconnaissance de droits "objectifs"(?) puissent automatiquement (sic!) avoir une influence sur notre conception du droit (Law) » n'est pas nôtre. Elle provient, de toute évidence, de ce que l'auteur ne comprend pas clairement la question transcendantale du droit, celle des possibilités théoriques sans lesquelles les revendications faites de nos jours en référence aux droits n'auraient aucun sens. C'est cette question transcendantale, pour encore une fois le souligner, que nous appelons criticisme.

Contrairement à ce que pense F. Blais il ne s'agit nullement d'une théorie naturaliste du droit dont on peut bien interroger aujourd'hui l'utilité et même la signification, mais bien d'une réflexion sur les conditions même d'une théorie normative que nous n'avons pas cherchè à faire passer pour une theorie naturaliste du droit. Nulle part ailleurs, dans l'ouvrage nous ne nous réclamons « naturalistes juridiques », un concept dont, il faut le remarquer au passage, le sens ne nous serait pas d'une grande évidence. Peut-être l'auteur en décrypte-t-il lui-même plus facilement le sens et le secret que nous ne saurions le faire. Pour peu qu'on comprenne la signification du criticisme qui est 
défendu ou qu'on se donne la peine de lire attentivement la définition que nous en donnons, (ce qui n'est pourtant pas trop difficile à réaliser pour une personne qui relève les moindres distinctions analytiques de Hart ou de Weinreb) on s'épargne de bien fâcheuses et grossières méprises.

Par criticisme juridique nous entendons ici, non pas tant les doctrines constituées chez Kant et Fichte, qu'un type de philosophie du droit, défini par un certain nombre d'options fondamentales, où nous parait résider la possibilité de mener à bien, dans un cadre intellectuellement moderne, un évitement de l'historicisme et du positivisme (p. 367).

C'est d'ailleurs ce qui justifie l'adoption du titre : « retour à Kant ou transformation de la philosophie critique ». F. Blais n'en a pas très bien saisi la portée qui veut avant tout montrer comment le criticisme, une fois notre définition adoptée «se trouve retravaillé par la philosophie juridico-politique contemporaine [...] à la faveur de problématiques sensiblement différentes de celles qui dominaient l'époque de Kant ou de Fichte. » (p. 368) Mais là également, la légèreté de la polémique s'est encore une fois, hélas, substituée à l'exigence de l'effort intellectuel. On parle d'« utilisation précipitée » d'un «exercice peu convaincant pour plusieurs » avant de répéter ce que nous disons : aussi bien la théorie de la justice de Rawls que l'éthique de la discussion d'Apel et de Habermas, (plus récemment encore Legitimität und Geltung de Habermas) sont, chacun à sa manière, tributaire de la philosophie kantienne. Non seulement elles se gardent bien d'être une répétition stérile de Kant, mais elles évitent le positivisme et l'historicisme. Ou alors l'auteur ne connaît pas très bien l'éthique de la discussion et ce qu'elle doit à Kant, ce qui devrait faire baisser le ton à l'auteur, ou alors il ignore la portée de ce qu'il dit lui-même et le sens proprement philosophiquement qu'on peut accorder à sa propre affirmation, à savoir : « l'essentiel de l'entreprise de Rawls a été de montrer qu'il nous était possible de nous entendre sur des principes moraux substantiels qui seraient une réponse à la fois aux conceptions métaphysiques des théories antérieures et à l'intuitionnisme. C'est tout le contraire défendu par les auteurs. $\gg$ Naturellement l'auteur se garde bien d'indiquer en quoi nous disons tout le contraire. Cela lui demandera trop d'effort et l'obligera à la démonstration. Par où l'on voit qu'au lieu de chercher à comprendre, en termes philosophiques, ce que cette tentative de Rawls signifie, F. Blais a préféré dire qu'elle est exactement le contraire du criticisme juridique. Mais alors comment qualifier une entreprise de fondation du droit qui évite et le positivisme et l'historicisme autrement qu'une entreprise criticiste, du moins la version que nous pouvons en retenir encore aujourd'hui ? Or, si aussi bien Rawls (ce que l'auteur recommande expressément à notre attention) que Habermas se réclament de Kant, qu'est-ce qui interdit de parler de leur entreprise en termes de « retour à Kant » au-delà même des divergences qui peuvent opposer les deux théories? Bref: pourquoi ne pas voir dans la théorie du sujet de l'éthique de la discussion la reconstruction de la pensée kantienne de la subjectivité pratique par trop dualiste et trop métaphysique et donc quasiment incompatible avec les exigences theoriques d'une argumentation philosophique contemporaine? Et si l'on s'accorde à y voir une tentative d'éviter une conception métaphysique du sujet (peu importe d'ailleurs le sens que Rawls en retient, puisqu'il ne définit nulle part le concept) pourquoi ne pourrait-on pas rapprocher justement les deux auteurs sur ce point? 
Ce que révèle pourtant une lecture attentive de Philosophie du droit c'est précisément comment ces auteurs, Rawls et Habermas, ont su reconstruire une perspective kantienne compatible avec les exigences d'une argumentation philosophique contemporaine, perspective qui constitue une réponse aux théories métaphysiques et non-cognitivistes et même aux théories philosophiques hostiles à la pensée du sujet et à la perspective philosophique normative du droit. Or, précisément, il se trouve que ces théories philosophiques qui récusent la pensée du sujet s'identifient aux formes de positivisme et d'historicisme dont nous faisons la critique. Il nous a donc semblé judicieux de donner deux illustrations contemporaines de réaménagement du dispositif théorique kantien dans la tentative de penser le droit ou l'éthique. François Blais, redisons-le, n'a pas tout à fait saisi l'objectif puisqu'il prend Philosophie du droit pour une théorie naturaliste du droit et se demande s'il s'agit d'une défense des droits substantiels. Ce n'est ni l'une ni l'autre, mais avec un peu d'effort encore, il saura distinguer une question des possibilités d'une pensée du droit aujourd'hui de ce qu'il est lui-même peut-être seul à comprendre, ả savoir une théorie naturaliste du droit. Seule une lecture où la précipation se fait complice de la mauvaise foi que renforce l'ignorance des enjeux réels de la controverse entre le positivisme juridique et du droit naturel peut parvenir aux conclusions de F. Blais sur Philosophie du droit. Une bonne intelligence de l'objectif de l'ouvrage pourra lui servir d'orientation pour sa prochaine critique.

Département de philosophie

Université de Montréal-Paris IV 